\title{
Best proximity point theorems for rational proximal contractions
}

\author{
Hemant Kumar Nashine ${ }^{1}$, Poom Kumam ${ }^{2 *}$ and Calogero Vetro ${ }^{3}$
}

${ }^{*}$ Correspondence:

poom.kum@kmutt.ac.th

${ }^{2}$ Department of Mathematics,

Faculty of Science, King Mongkut's

University of Technology Thonburi (KMUTT), Bangkok, 10140, Thailand

Full list of author information is

available at the end of the article

\begin{abstract}
We provide sufficient conditions which warrant the existence and uniqueness of the best proximity point for two new types of contractions in the setting of metric spaces. The presented results extend, generalize and improve some known results from best proximity point theory and fixed-point theory. We also give some examples to illustrate and validate our definitions and results.
\end{abstract}

MSC: 41A65; 46B20; 47H10

Keywords: best proximity point; contraction; fixed point; generalized proximal contraction; optimal approximate solution

\section{Introduction}

Let $(\mathcal{X}, d)$ be a metric space and $\mathcal{T}$ be a self-mapping defined on a subset of $\mathcal{X}$. In this setting, the fixed-point theory is an important tool for solving equations of the kind $\mathcal{T} x=$ $x$, whose solutions are the fixed points of the mapping $\mathcal{T}$. On the other hand, if $\mathcal{T}$ is not a self-mapping, say $\mathcal{T}: \mathcal{A} \rightarrow \mathcal{B}$ where $\mathcal{A}$ and $\mathcal{B}$ are nonempty subsets of $\mathcal{X}$, then $\mathcal{T}$ does not necessarily have a fixed point. Consequently, the equation $\mathcal{T} x=x$ could have no solutions, and in this case, it is of a certain interest to determine an element $x$ that is in some sense closest to $\mathcal{T} x$. Thus, we can say that the aim of the best proximity point theorems is to provide sufficient conditions to solve a minimization problem. In view of the fact that $d(x, \mathcal{T} x)$ is at least $d(\mathcal{A}, \mathcal{B}):=\inf \{d(x, y): x \in \mathcal{A}$ and $y \in \mathcal{B}\}$, a best proximity point theorem concerns the global minimum of the real valued function $x \rightarrow d(x, \mathcal{T} x)$, that is, an indicator of the error involved for an approximate solution of the equation $\mathcal{T} x=x$, by complying the condition $d(x, \mathcal{T} x)=d(\mathcal{A}, \mathcal{B})$. The notation of best proximity point is introduced in [1] but one of the most interesting results in this direction is due to Fan [2] and can be stated as follows.

Theorem 1.1 Let $\mathcal{K}$ be a nonempty, compact and convex subset of a normed space $\mathcal{E}$. Then for any continuous mapping $\mathcal{T}: \mathcal{K} \rightarrow \mathcal{E}$, there exists $x \in \mathcal{K}$ with $\|x-\mathcal{T} x\|=\inf _{y \in \mathcal{K}}\|\mathcal{T} x-y\|$.

Some generalizations and extensions of this theorem appeared in the literature by Prolla [3], Reich [4], Sehgal and Singh [5, 6], Vetrivel et al. [7] and others. It turns out that many of the contractive conditions which are investigated for fixed points ensure the existence of best proximity points. Some results of this kind are obtained in [1,5-40]. Note that the authors often, in proving these results, assume restrictive compactness hypotheses on the domain and codomain of the involved nonself-mapping. Inspired by [29], we

C) 2013 Nashine et al.; licensee Springer. This is an Open Access article distributed under the terms of the Creative Commons Attribution License (http://creativecommons.org/licenses/by/2.0), which permits unrestricted use, distribution, and reproduction in any medium, provided the original work is properly cited. 
consider these hypotheses too restrictive in dealing with proximal contractions and so we prove that the compactness hypotheses can be successfully replaced by standard completeness hypotheses. Following this idea, we propose a new type of condition to study the existence and uniqueness of the best proximity point of a nonself-mapping by assuming both compactness hypotheses and standard completeness hypotheses. Precisely, we introduce the notions of rational proximal contractions of the first and second kinds, then we establish some corresponding best proximity point theorems for such contractions. Our definitions include some earlier definitions as special cases. In particular, the presented theorems contain the results given in [29].

\section{Preliminaries}

In this section, we give some basic notations and definitions that will be used in the sequel.

Let $(\mathcal{X}, d)$ be a metric space, $\mathcal{A}$ and $\mathcal{B}$ be two nonempty subsets of $\mathcal{X}$ and $\mathcal{T}: \mathcal{A} \rightarrow \mathcal{B}$ be a nonself-mapping. We denote by $B_{\text {est }}(\mathcal{T})$ the set of all best proximity points of $\mathcal{T}$, that is,

$$
B_{\text {est }}(\mathcal{T}):=\{x \in \mathcal{A} \text { such that } d(x, \mathcal{T} x)=d(\mathcal{A}, \mathcal{B})\}
$$

Also, let

$$
\mathcal{A}_{0}:=\{x \in \mathcal{A}: d(x, y)=d(\mathcal{A}, \mathcal{B}) \text { for some } y \in \mathcal{B}\}
$$

and

$$
\mathcal{B}_{0}:=\{y \in \mathcal{B}: d(x, y)=d(\mathcal{A}, \mathcal{B}) \text { for some } x \in \mathcal{A}\}
$$

Sufficient conditions to ensure that $\mathcal{A}_{0}$ and $\mathcal{B}_{0}$ are nonempty are given in [41]. Also, observe that if $\mathcal{A}$ and $\mathcal{B}$ are closed subsets of a normed linear space such that $d(\mathcal{A}, \mathcal{B})>0$, then $\mathcal{A}_{0}$ and $\mathcal{B}_{0}$ are contained in the boundaries of $\mathcal{A}$ and $\mathcal{B}$, respectively; see [27].

Now, we give sequentially two definitions that are essential to state and prove our main results.

Definition 2.1 Let $(\mathcal{X}, d)$ be a metric space and $\mathcal{A}$ and $\mathcal{B}$ be two nonempty subsets of $\mathcal{X}$. Then $\mathcal{T}: \mathcal{A} \rightarrow \mathcal{B}$ is said to be a rational proximal contraction of the first kind if there exist nonnegative real numbers $\alpha, \beta, \gamma, \delta$ with $\alpha+\beta+2 \gamma+2 \delta<1$, such that the conditions

$$
d\left(u_{1}, \mathcal{T} x_{1}\right)=d(\mathcal{A}, \mathcal{B}) \quad \text { and } \quad d\left(u_{2}, \mathcal{T} x_{2}\right)=d(\mathcal{A}, \mathcal{B})
$$

imply that

$$
\begin{aligned}
d\left(u_{1}, u_{2}\right) \leq & \alpha d\left(x_{1}, x_{2}\right)+\frac{\beta\left[1+d\left(x_{1}, u_{1}\right)\right] d\left(x_{2}, u_{2}\right)}{1+d\left(x_{1}, x_{2}\right)}+\gamma\left[d\left(x_{1}, u_{1}\right)+d\left(x_{2}, u_{2}\right)\right] \\
& +\delta\left[d\left(x_{1}, u_{2}\right)+d\left(x_{2}, u_{1}\right)\right]
\end{aligned}
$$

for all $u_{1}, u_{2}, x_{1}, x_{2} \in \mathcal{A}$.

Note that, if $\beta=0$, then from (1) we get the definition of the generalized proximal contraction of the first kind with $\alpha+2 \gamma+2 \delta<1$; see [29]. 
Moreover, if $\mathcal{T}$ is a self-mapping on $\mathcal{A}$, then the requirement in Definition 2.1 reduces to the following generalized contractive condition of rational type useful in establishing a fixed-point theorem:

$$
\begin{aligned}
d\left(\mathcal{T} x_{1}, \mathcal{T} x_{2}\right) \leq & \alpha d\left(x_{1}, x_{2}\right)+\frac{\beta\left[1+d\left(x_{1}, \mathcal{T} x_{1}\right)\right] d\left(x_{2}, \mathcal{T} x_{2}\right)}{1+d\left(x_{1}, x_{2}\right)}+\gamma\left[d\left(x_{1}, \mathcal{T} x_{1}\right)+d\left(x_{2}, \mathcal{T} x_{2}\right)\right] \\
& +\delta\left[d\left(x_{1}, \mathcal{T} x_{2}\right)+d\left(x_{2}, \mathcal{T} x_{1}\right)\right]
\end{aligned}
$$

Definition 2.2 Let $(\mathcal{X}, d)$ be a metric space and $\mathcal{A}$ and $\mathcal{B}$ be two nonempty subsets of $\mathcal{X}$. Then $\mathcal{T}: \mathcal{A} \rightarrow \mathcal{B}$ is said to be a rational proximal contraction of the second kind if there exist nonnegative real numbers $\alpha, \beta, \gamma, \delta$ with $\alpha+\beta+2 \gamma+2 \delta<1$ such that the conditions

$$
d\left(u_{1}, \mathcal{T} x_{1}\right)=d(\mathcal{A}, \mathcal{B}) \quad \text { and } \quad d\left(u_{2}, \mathcal{T} x_{2}\right)=d(\mathcal{A}, \mathcal{B})
$$

imply that

$$
\begin{aligned}
& d\left(\mathcal{T} u_{1}, \mathcal{T} u_{2}\right) \\
& \leq \alpha d\left(\mathcal{T} x_{1}, \mathcal{T} x_{2}\right)+\frac{\beta\left[1+d\left(\mathcal{T} x_{1}, \mathcal{T} u_{1}\right)\right] d\left(\mathcal{T} x_{2}, \mathcal{T} u_{2}\right)}{1+d\left(\mathcal{T} x_{1}, \mathcal{T} x_{2}\right)} \\
& \quad+\gamma\left[d\left(\mathcal{T} x_{1}, \mathcal{T} u_{1}\right)+d\left(\mathcal{T} x_{2}, \mathcal{T} u_{2}\right)\right]+\delta\left[d\left(\mathcal{T} x_{1}, \mathcal{T} u_{2}\right)+d\left(\mathcal{T} x_{2}, \mathcal{T} u_{1}\right)\right]
\end{aligned}
$$

for all $u_{1}, u_{2}, x_{1}, x_{2} \in \mathcal{A}$.

Note that, if $\beta=0$, then from (2) we get the definition of the generalized proximal contraction of the second kind with $\alpha+2 \gamma+2 \delta<1$, see [29].

The following example illustrates that a rational proximal contraction of the second kind is not necessarily a rational proximal contraction of the first kind. Therefore, both Definitions 2.1 and 2.2 are consistent.

Example 2.1 Let $\mathcal{X}=\mathbb{R} \times \mathbb{R}$ endowed with the usual metric

$$
d\left(\left(x_{1}, x_{2}\right),\left(y_{1}, y_{2}\right)\right)=\sqrt{\left(x_{1}-y_{1}\right)^{2}+\left(x_{2}-y_{2}\right)^{2}},
$$

for all $\left(x_{1}, x_{2}\right),\left(y_{1}, y_{2}\right) \in \mathbb{R} \times \mathbb{R}$. Define $\mathcal{A}:=\{(x, 1): x \in \mathbb{R}\}$ and $\mathcal{B}:=\{(x,-1): x \in \mathbb{R}\}$. Also define $\mathcal{T}: \mathcal{A} \rightarrow \mathcal{B}$ by

$$
\mathcal{T}((x, 1))= \begin{cases}(-1,-1) & \text { if } x \text { is rational } \\ (1,-1) & \text { otherwise }\end{cases}
$$

Then $d(\mathcal{A}, \mathcal{B})=2$ and $\mathcal{T}$ is a rational proximal contraction of the second kind but not a rational proximal contraction of the first kind. Indeed, using Definition 2.2 and after routine calculations, one can show that the left-hand side of inequality (2) is equal to 0 . On the other hand, using Definition 2.1 and after routine calculations, one can show that the left-hand side of inequality (1) is equal to 2 and so inequality (1) is not satisfied for all nonnegative real numbers $\alpha, \beta, \gamma, \delta$ with $\alpha+\beta+2 \gamma+2 \delta<1$. 
It is well known that the notion of approximative compactness plays an important role in the theory of approximation [12]. In particular, the notion of an approximatively compact set was introduced by Efimov and Stechkin [16] and the properties of approximatively compact sets have been largely studied. The boundendly compact sets that are the sets whose intersection with any closed ball is compact are useful examples of approximatively compact sets. It is shown in [14] that in every infinite-dimensional separable Banach space there exists a bounded approximatively compact set, which is not compact.

Remark 2.1 Since $(\mathcal{X}, d)$ is a metric space, the bounded compactness of a set is equivalent to its closure and the possibility of selecting from any bounded sequence contained in it a converging subsequence.

Here, for our further use, we give the following definition.

Definition 2.3 Let $(\mathcal{X}, d)$ be a metric space and $\mathcal{A}$ and $\mathcal{B}$ be two nonempty subsets of $\mathcal{X}$. Then $\mathcal{B}$ is said to be approximatively compact with respect to $\mathcal{A}$ if every sequence $\left\{y_{n}\right\}$ of $\mathcal{B}$, satisfying the condition $d\left(x, y_{n}\right) \rightarrow d(x, \mathcal{B})$ for some $x$ in $\mathcal{A}$, has a convergent subsequence.

Obviously, any set is approximatively compact with respect to itself.

\section{Rational proximal contractions}

Our first main result is the following best proximity point theorem for a rational proximal contraction of the first kind.

Theorem 3.1 Let $(\mathcal{X}, d)$ be a complete metric space and $\mathcal{A}$ and $\mathcal{B}$ be two nonempty, closed subsets of $\mathcal{X}$ such that $\mathcal{B}$ is approximatively compact with respect to $\mathcal{A}$. Assume that $\mathcal{A}_{0}$ and $\mathcal{B}_{0}$ are nonempty and $\mathcal{T}: \mathcal{A} \rightarrow \mathcal{B}$ is a nonself-mapping such that:

(a) $\mathcal{T}$ is a rational proximal contraction of the first kind;

(b) $\mathcal{T}\left(\mathcal{A}_{0}\right) \subseteq \mathcal{B}_{0}$.

Then there exists $x \in \mathcal{A}$ such that $B_{\text {est }}(\mathcal{T})=\{x\}$. Further, for any fixed $x_{0} \in \mathcal{A}_{0}$, the sequence $\left\{x_{n}\right\}$, defined by $d\left(x_{n+1}, \mathcal{T} x_{n}\right)=d(\mathcal{A}, \mathcal{B})$, converges to $x$.

Proof Let $x_{0} \in \mathcal{A}_{0}$ (such a point there exists since $\mathcal{A}_{0} \neq \emptyset$ ). Since $\mathcal{T}\left(\mathcal{A}_{0}\right) \subseteq \mathcal{B}_{0}$, then by the definition of $\mathcal{B}_{0}$, there exists $x_{1} \in \mathcal{A}_{0}$ such that $d\left(x_{1}, \mathcal{T} x_{0}\right)=d(\mathcal{A}, \mathcal{B})$. Again, since $\mathcal{T} x_{1} \in \mathcal{B}_{0}$, it follows that there is $x_{2} \in \mathcal{A}_{0}$ such that $d\left(x_{2}, \mathcal{T} x_{1}\right)=d(\mathcal{A}, \mathcal{B})$. Continuing this process, we can construct a sequence $\left\{x_{n}\right\}$ in $\mathcal{A}_{0}$, such that

$$
d\left(x_{n+1}, \mathcal{T} x_{n}\right)=d(\mathcal{A}, \mathcal{B})
$$

for every nonnegative integer $n$. Using the fact that $\mathcal{T}$ is a rational proximal contraction of the first kind, we have

$$
\begin{aligned}
d\left(x_{n}, x_{n+1}\right) \leq & \alpha d\left(x_{n-1}, x_{n}\right)+\frac{\beta\left[1+d\left(x_{n-1}, x_{n}\right)\right] d\left(x_{n}, x_{n+1}\right)}{1+d\left(x_{n-1}, x_{n}\right)}+\gamma\left[d\left(x_{n-1}, x_{n}\right)+d\left(x_{n}, x_{n+1}\right)\right] \\
& +\delta d\left(x_{n-1}, x_{n+1}\right) \\
\leq & \alpha d\left(x_{n-1}, x_{n}\right)+\beta d\left(x_{n}, x_{n+1}\right)+\gamma\left[d\left(x_{n-1}, x_{n}\right)+d\left(x_{n}, x_{n+1}\right)\right] \\
& +\delta\left[d\left(x_{n-1}, x_{n}\right)+d\left(x_{n}, x_{n+1}\right)\right] .
\end{aligned}
$$


It follows that

$$
d\left(x_{n}, x_{n+1}\right) \leq k d\left(x_{n-1}, x_{n}\right),
$$

where $k=\frac{\alpha+\gamma+\delta}{1-\beta-\gamma-\delta}<1$. Therefore, $\left\{x_{n}\right\}$ is a Cauchy sequence and, since $(\mathcal{X}, d)$ is complete and $\mathcal{A}$ is closed, the sequence $\left\{x_{n}\right\}$ converges to some $x \in \mathcal{A}$.

Moreover, we have

$$
\begin{aligned}
d(x, \mathcal{B}) & \leq d\left(x, \mathcal{T} x_{n}\right) \\
& \leq d\left(x, x_{n+1}\right)+d\left(x_{n+1}, \mathcal{T} x_{n}\right) \\
& =d\left(x, x_{n+1}\right)+d(\mathcal{A}, \mathcal{B}) \\
& \leq d\left(x, x_{n+1}\right)+d(x, \mathcal{B}) .
\end{aligned}
$$

Taking the limit as $n \rightarrow+\infty$, we get $d\left(x, \mathcal{T} x_{n}\right) \rightarrow d(x, \mathcal{B})$. Since $\mathcal{B}$ is approximatively compact with respect to $\mathcal{A}$, then the sequence $\left\{\mathcal{T} x_{n}\right\}$ has a subsequence $\left\{\mathcal{T} x_{n_{k}}\right\}$ that converges to some $y \in \mathcal{B}$. Therefore,

$$
d(x, y)=\lim _{k \rightarrow+\infty} d\left(x_{n_{k}+1}, \mathcal{T} x_{n_{k}}\right)=d(\mathcal{A}, \mathcal{B})
$$

and hence $x$ must be in $\mathcal{A}_{0}$. Since $\mathcal{T}\left(\mathcal{A}_{0}\right) \subseteq \mathcal{B}_{0}$, then $d(u, \mathcal{T} x)=d(\mathcal{A}, \mathcal{B})$ for some $u \in \mathcal{A}$. Again, using the fact that $\mathcal{T}$ is a rational proximal contraction of the first kind, we get

$$
\begin{aligned}
d\left(u, x_{n+1}\right) \leq & \alpha d\left(x, x_{n}\right)+\frac{\beta[1+d(x, u)] d\left(x_{n}, x_{n+1}\right)}{1+d\left(x, x_{n}\right)}+\gamma\left[d(x, u)+d\left(x_{n}, x_{n+1}\right)\right] \\
& +\delta\left[d\left(x, x_{n+1}\right)+d\left(x_{n}, u\right)\right] .
\end{aligned}
$$

Taking the limit as $n \rightarrow+\infty$, we have

$$
d(u, x) \leq(\gamma+\delta) d(u, x)
$$

which implies $x=u$, since $\gamma+\delta<1$. Thus, it follows that

$$
d(x, \mathcal{T} x)=d(u, \mathcal{T} x)=d(\mathcal{A}, \mathcal{B})
$$

that is, $x \in B_{\text {est }}(\mathcal{T})$. Now, to prove the uniqueness of the best proximity point $\left(i . e ., B_{\text {est }}(\mathcal{T})\right.$ is singleton), assume that $z$ is another best proximity point of $\mathcal{T}$ so that

$$
d(z, \mathcal{T} z)=d(\mathcal{A}, \mathcal{B})
$$

Since $\mathcal{T}$ is a rational proximal contraction of the first kind, we have

$$
d(x, z) \leq \alpha d(x, z)+\frac{\beta[1+d(x, x)] d(z, z)}{1+d(x, z)}+\gamma[d(x, x)+d(z, z)]+\delta[d(x, z)+d(z, x)]
$$

which implies

$$
d(x, z) \leq(\alpha+2 \delta) d(x, z)
$$


It follows immediately that $x=z$, since $\alpha+2 \delta<1$. Hence, $\mathcal{T}$ has a unique best proximity point.

As consequences of the Theorem 3.1, we state the following corollaries.

Corollary 3.1 Let $(\mathcal{X}, d)$ be a complete metric space and $\mathcal{A}$ and $\mathcal{B}$ be two nonempty, closed subsets of $\mathcal{X}$ such that $\mathcal{B}$ is approximatively compact with respect to $\mathcal{A}$. Assume that $\mathcal{A}_{0}$ and $\mathcal{B}_{0}$ are nonempty and $\mathcal{T}: \mathcal{A} \rightarrow \mathcal{B}$ is a nonself-mapping such that:

(a) $\mathcal{T}$ is a generalized proximal contraction of the first kind, with $\alpha+2 \gamma+2 \delta<1$;

(b) $\mathcal{T}\left(\mathcal{A}_{0}\right) \subseteq \mathcal{B}_{0}$.

Then, there exists $x \in \mathcal{A}$ such that $B_{\text {est }}(\mathcal{T})=\{x\}$. Further, for any fixed $x_{0} \in \mathcal{A}_{0}$, the sequence $\left\{x_{n}\right\}$, defined by $d\left(x_{n+1}, \mathcal{T} x_{n}\right)=d(\mathcal{A}, \mathcal{B})$, converges to the best proximity point $x$.

Corollary 3.2 Let $(\mathcal{X}, d)$ be a complete metric space and $\mathcal{A}$ and $\mathcal{B}$ be two nonempty, closed subsets of $\mathcal{X}$ such that $\mathcal{B}$ is approximatively compact with respect to $\mathcal{A}$. Assume that $\mathcal{A}_{0}$ and $\mathcal{B}_{0}$ are nonempty and $\mathcal{T}: \mathcal{A} \rightarrow \mathcal{B}$ is a nonself-mapping such that:

(a) There exists a nonnegative real number $\alpha<1$ such that, for all $u_{1}, u_{2}, x_{1}, x_{2}$ in $\mathcal{A}$, the conditions $d\left(u_{1}, \mathcal{T} x_{1}\right)=d(\mathcal{A}, \mathcal{B})$ and $d\left(u_{2}, \mathcal{T} x_{2}\right)=d(\mathcal{A}, \mathcal{B})$ imply that $d\left(u_{1}, u_{2}\right) \leq \alpha d\left(x_{1}, x_{2}\right)$

(b) $\mathcal{T}\left(\mathcal{A}_{0}\right) \subseteq \mathcal{B}_{0}$.

Then there exists $x \in \mathcal{A}$ such that $B_{\text {est }}(\mathcal{T})=\{x\}$. Further, for any fixed $x_{0} \in \mathcal{A}_{0}$, the sequence $\left\{x_{n}\right\}$, defined by $d\left(x_{n+1}, \mathcal{T} x_{n}\right)=d(\mathcal{A}, \mathcal{B})$, converges to the best proximity point $x$.

The following fixed-point result can be considered as a special case of the Theorem 3.1, when $\mathcal{T}$ is a self-mapping.

Corollary 3.3 Let $(\mathcal{X}, d)$ be a complete metric space and $\mathcal{T}$ be a self-mapping on $\mathcal{X}$. Assume that there exist nonnegative real numbers $\alpha, \beta, \gamma, \delta$ with $\alpha+\beta+2 \gamma+2 \delta<1$ such that

$$
\begin{aligned}
d\left(\mathcal{T} x_{1}, \mathcal{T} x_{2}\right) \leq & \alpha d\left(x_{1}, x_{2}\right)+\frac{\beta\left[1+d\left(x_{1}, \mathcal{T} x_{2}\right)\right] d\left(x_{2}, \mathcal{T} x_{2}\right)}{1+d\left(x_{1}, x_{2}\right)}+\gamma\left[d\left(x_{1}, \mathcal{T} x_{1}\right)+d\left(x_{2}, \mathcal{T} x_{2}\right)\right] \\
& +\delta\left[d\left(x_{1}, \mathcal{T} x_{2}\right)+d\left(x_{2}, \mathcal{T} x_{1}\right)\right]
\end{aligned}
$$

for all $x_{1}, x_{2} \in \mathcal{X}$. Then the mapping $\mathcal{T}$ has a unique fixed point.

Remark 3.1 Note that the Corollary 3.3 is a proper extension of the contraction mapping principle [13] because the continuity of the mapping $\mathcal{T}$ is not required. It is well known that a contraction mapping must be continuous.

Now, we state and prove a best proximity point theorem for a rational proximal contraction of the second kind.

Theorem 3.2 Let $(\mathcal{X}, d)$ be a complete metric space and $\mathcal{A}$ and $\mathcal{B}$ be two nonempty, closed subsets of $\mathcal{X}$ such that $\mathcal{A}$ is approximatively compact with respect to $\mathcal{B}$. Assume that $\mathcal{A}_{0}$ and $\mathcal{B}_{0}$ are nonempty and $\mathcal{T}: \mathcal{A} \rightarrow \mathcal{B}$ is a nonself-mapping such that:

(a) $\mathcal{T}$ is a continuous rational proximal contraction of the second kind;

(b) $\mathcal{T}\left(\mathcal{A}_{0}\right) \subseteq \mathcal{B}_{0}$ 
Then there exists $x \in B_{\text {est }}(\mathcal{T})$ and for any fixed $x_{0} \in \mathcal{A}_{0}$, the sequence $\left\{x_{n}\right\}$, defined by $d\left(x_{n+1}, \mathcal{T} x_{n}\right)=d(\mathcal{A}, \mathcal{B})$, converges to $x$, and $\mathcal{T} x=\mathcal{T} z$ for all $x, z \in B_{\text {est }}(\mathcal{T})$.

Proof Following the same lines of the proof of the Theorem 3.1, it is possible to construct a sequence $\left\{x_{n}\right\}$ in $\mathcal{A}_{0}$ such that

$$
d\left(x_{n+1}, \mathcal{T} x_{n}\right)=d(\mathcal{A}, \mathcal{B})
$$

for every nonnegative integer $n$. Using the fact that $\mathcal{T}$ is a rational proximal contraction of the second kind, we have

$$
\begin{aligned}
& d\left(\mathcal{T} x_{n}, \mathcal{T} x_{n+1}\right) \\
& \leq \alpha d\left(\mathcal{T} x_{n-1}, \mathcal{T} x_{n}\right)+\frac{\beta\left[1+d\left(\mathcal{T} x_{n-1}, \mathcal{T} x_{n}\right)\right] d\left(\mathcal{T} x_{n}, \mathcal{T} x_{n+1}\right)}{1+d\left(\mathcal{T} x_{n-1}, \mathcal{T} x_{n}\right)} \\
& \quad+\gamma\left[d\left(\mathcal{T} x_{n-1}, \mathcal{T} x_{n}\right)+d\left(\mathcal{T} x_{n}, \mathcal{T} x_{n+1}\right)\right]+\delta d\left(\mathcal{T} x_{n-1}, \mathcal{T} x_{n+1}\right) \\
& \leq \alpha d\left(\mathcal{T} x_{n-1}, \mathcal{T} x_{n}\right)+\beta d\left(\mathcal{T} x_{n}, \mathcal{T} x_{n+1}\right)+\gamma\left[d\left(\mathcal{T} x_{n-1}, \mathcal{T} x_{n}\right)+d\left(\mathcal{T} x_{n}, \mathcal{T} x_{n+1}\right)\right] \\
& \quad+\delta\left[d\left(\mathcal{T} x_{n-1}, \mathcal{T} x_{n}\right)+d\left(\mathcal{T} x_{n}, \mathcal{T} x_{n+1}\right)\right] .
\end{aligned}
$$

It follows that

$$
d\left(\mathcal{T} x_{n}, \mathcal{T} x_{n+1}\right) \leq k d\left(\mathcal{T} x_{n-1}, \mathcal{T} x_{n}\right)
$$

where $k=\frac{\alpha+\gamma+\delta}{1-\beta-\gamma-\delta}<1$. Therefore, $\left\{\mathcal{T} x_{n}\right\}$ is a Cauchy sequence and, since $(\mathcal{X}, d)$ is complete, then the sequence $\left\{\mathcal{T} x_{n}\right\}$ converges to some $y \in \mathcal{B}$.

Moreover, we have

$$
\begin{aligned}
d(y, \mathcal{A}) & \leq d\left(y, x_{n+1}\right) \leq d\left(y, \mathcal{T} x_{n}\right)+d\left(\mathcal{T} x_{n}, x_{n+1}\right) \\
& =d\left(y, \mathcal{T} x_{n}\right)+d(\mathcal{A}, \mathcal{B}) \leq d\left(y, \mathcal{T} x_{n}\right)+d(y, \mathcal{A}) .
\end{aligned}
$$

Taking the limit as $n \rightarrow+\infty$, we get $d\left(y, x_{n}\right) \rightarrow d(y, \mathcal{A})$. Since $\mathcal{A}$ is approximatively compact with respect to $\mathcal{B}$, then the sequence $\left\{x_{n}\right\}$ has a subsequence $\left\{x_{n_{k}}\right\}$ converging to some $x \in \mathcal{A}$. Now, using the continuity of $\mathcal{T}$, we obtain that

$$
d(x, \mathcal{T} x)=\lim _{k \rightarrow+\infty} d\left(x_{n_{k}+1}, \mathcal{T} x_{n_{k}}\right)=d(\mathcal{A}, \mathcal{B})
$$

that is, $x \in B_{\text {est }}(\mathcal{T})$. Finally, to prove the last assertion of the present theorem, assume that $z$ is another best proximity point of $\mathcal{T}$ so that

$$
d(z, \mathcal{T} z)=d(\mathcal{A}, \mathcal{B})
$$

Since $\mathcal{T}$ is a rational proximal contraction of the second kind, we have

$$
\begin{aligned}
d(\mathcal{T} x, \mathcal{T} z) \leq & \alpha d(\mathcal{T} x, \mathcal{T} z)+\frac{\beta[1+d(\mathcal{T} x, \mathcal{T} x)] d(\mathcal{T} z, \mathcal{T} z)}{1+d(\mathcal{T} x, \mathcal{T} z)}+\gamma[d(\mathcal{T} x, \mathcal{T} x)+d(\mathcal{T} z, \mathcal{T} z)] \\
& +\delta[d(\mathcal{T} x, \mathcal{T} z)+d(\mathcal{T} z, \mathcal{T} x)]
\end{aligned}
$$


which implies

$$
d(\mathcal{T} x, \mathcal{T} z) \leq(\alpha+2 \delta) d(\mathcal{T} x, \mathcal{T} z)
$$

It follows immediately that $\mathcal{T} x=\mathcal{T} z$, since $\alpha+2 \delta<1$.

As consequences of the Theorem 3.2, we state the following corollaries.

Corollary 3.4 Let $(\mathcal{X}, d)$ be a complete metric space and $\mathcal{A}$ and $\mathcal{B}$ be two nonempty, closed subsets of $\mathcal{X}$ such that $\mathcal{A}$ is approximatively compact with respect to $\mathcal{B}$. Assume that $\mathcal{A}_{0}$ and $\mathcal{B}_{0}$ are nonempty and $\mathcal{T}: \mathcal{A} \rightarrow \mathcal{B}$ is a nonself-mapping such that:

(a) $\mathcal{T}$ is a continuous generalized proximal contraction of the second kind, with $\alpha+2 \gamma+2 \delta<1$

(b) $\mathcal{T}\left(\mathcal{A}_{0}\right) \subseteq \mathcal{B}_{0}$.

Then, there exists $x \in B_{\text {est }}(\mathcal{T})$ and for any fixed $x_{0} \in \mathcal{A}_{0}$, the sequence $\left\{x_{n}\right\}$, defined by $d\left(x_{n+1}, \mathcal{T} x_{n}\right)=d(\mathcal{A}, \mathcal{B})$, converges to $x$. Further, $\mathcal{T} x=\mathcal{T} z$ for all $x, z \in B_{\text {est }}(\mathcal{T})$.

Corollary 3.5 Let $(\mathcal{X}, d)$ be a complete metric space and $\mathcal{A}$ and $\mathcal{B}$ be two nonempty, closed subsets of $\mathcal{X}$ such that $\mathcal{A}$ is approximatively compact with respect to $\mathcal{B}$. Assume that $\mathcal{A}_{0}$ and $\mathcal{B}_{0}$ are nonempty and $\mathcal{T}: \mathcal{A} \rightarrow \mathcal{B}$ is a nonself-mapping such that:

(a) There exists a nonnegative real number $\alpha<1$ such that, for all $u_{1}, u_{2}, x_{1}, x_{2}$ in $\mathcal{A}$, the conditions $d\left(u_{1}, \mathcal{T} x_{1}\right)=d(\mathcal{A}, \mathcal{B})$ and $d\left(u_{2}, \mathcal{T} x_{2}\right)=d(\mathcal{A}, \mathcal{B})$ imply that $d\left(\mathcal{T} u_{1}, \mathcal{T} u_{2}\right) \leq \alpha d\left(\mathcal{T} x_{1}, \mathcal{T} x_{2}\right)$

(b) $\mathcal{T}$ is continuous;

(c) $\mathcal{T}\left(\mathcal{A}_{0}\right) \subseteq \mathcal{B}_{0}$.

Then there exists $x \in B_{\text {est }}(\mathcal{T})$ and for any fixed $x_{0} \in \mathcal{A}_{0}$, the sequence $\left\{x_{n}\right\}$, defined by $d\left(x_{n+1}, \mathcal{T} x_{n}\right)=d(\mathcal{A}, \mathcal{B})$, converges to $x$. Further, $\mathcal{T} x=\mathcal{T} z$ for all $x, z \in B_{\text {est }}(\mathcal{T})$.

Remark 3.2 Note that in the Theorem 3.1 is not required the continuity of the mapping $\mathcal{T}$. On the contrary, the continuity of $\mathcal{T}$ is an hypothesis of the Theorem 3.2.

Our next theorem concerns a nonself-mapping that is a rational proximal contraction of the first kind as well as a rational proximal contraction of the second kind. In this theorem, we consider only a completeness hypothesis without assuming the continuity of the nonself-mapping.

Theorem 3.3 Let $(\mathcal{X}, d)$ be a complete metric space and $\mathcal{A}$ and $\mathcal{B}$ be two nonempty, closed subsets of $\mathcal{X}$. Assume that $\mathcal{A}_{0}$ and $\mathcal{B}_{0}$ are nonempty and $\mathcal{T}: \mathcal{A} \rightarrow \mathcal{B}$ is a nonself-mapping such that:

(a) $\mathcal{T}$ is a rational proximal contraction of the first and second kinds;

(b) $\mathcal{T}\left(\mathcal{A}_{0}\right) \subseteq \mathcal{B}_{0}$.

Then there exists a unique $x \in B_{\text {est }}(\mathcal{T})$. Further, for any fixed $x_{0} \in \mathcal{A}_{0}$, the sequence $\left\{x_{n}\right\}$, defined by $d\left(x_{n+1}, \mathcal{T} x_{n}\right)=d(\mathcal{A}, \mathcal{B})$, converges to $x$.

Proof Following the same lines of the proof of the Theorem 3.1, it is possible to construct a sequence $\left\{x_{n}\right\}$ in $\mathcal{A}_{0}$ such that

$$
d\left(x_{n+1}, \mathcal{T} x_{n}\right)=d(\mathcal{A}, \mathcal{B})
$$


for every nonnegative integer $n$. Also, using the same arguments in the proof of the Theorem 3.1, we deduce that the sequence $\left\{x_{n}\right\}$ is a Cauchy sequence, and hence converges to some $x \in \mathcal{A}$. Moreover, on the lines of the proof of the Theorem 3.2, we obtain that the sequence $\left\{\mathcal{T} x_{n}\right\}$ is a Cauchy sequence and hence converges to some $y \in \mathcal{B}$. Therefore, we have

$$
d(x, y)=\lim _{n \rightarrow+\infty} d\left(x_{n+1}, \mathcal{T} x_{n}\right)=d(\mathcal{A}, \mathcal{B})
$$

and hence $x$ must be in $\mathcal{A}_{0}$. Since $\mathcal{T}\left(\mathcal{A}_{0}\right) \subseteq \mathcal{B}_{0}$, then $d(u, \mathcal{T} x)=d(\mathcal{A}, \mathcal{B})$ for some $u \in \mathcal{A}$. Using the fact that $\mathcal{T}$ is a rational proximal contraction of the first kind, we get

$$
\begin{aligned}
d\left(u, x_{n+1}\right) \leq & \alpha d\left(x, x_{n}\right)+\frac{\beta[1+d(x, u)] d\left(x_{n}, x_{n+1}\right)}{1+d\left(x, x_{n}\right)} \\
& +\gamma\left[d(x, u)+d\left(x_{n}, x_{n+1}\right)\right]+\delta\left[d\left(x, x_{n+1}\right)+d\left(x_{n}, u\right)\right] .
\end{aligned}
$$

Taking the limit as $n \rightarrow+\infty$, we have

$$
d(u, x) \leq(\gamma+\delta) d(u, x)
$$

which implies that $x=u$, since $\gamma+\delta<1$. Thus, it follows that

$$
d(x, \mathcal{T} x)=d(u, \mathcal{T} x)=d(\mathcal{A}, \mathcal{B})
$$

that is, $x \in B_{\text {est }}(\mathcal{T})$. Again, following the same lines of the proof of the Theorem 3.1, we prove the uniqueness of the best proximity point of the mapping $\mathcal{T}$. To avoid repetitions, we omit the details.

Example 3.1 Let $\mathcal{X}=\mathbb{R}$ endowed with the usual metric $d(x, y)=|x-y|$, for all $x, y \in \mathcal{X}$. Define $\mathcal{A}=[-1,1]$ and $\mathcal{B}=[-3,-2] \cup[2,3]$. Then, $d(\mathcal{A}, \mathcal{B})=1, \mathcal{A}_{0}=\{-1,1\}$ and $\mathcal{B}_{0}=\{-2,2\}$. Also define $\mathcal{T}: \mathcal{A} \rightarrow \mathcal{B}$ by

$$
\mathcal{T} x= \begin{cases}2 & \text { if } x \text { is rational } \\ 3 & \text { otherwise }\end{cases}
$$

It is easy to show that $\mathcal{T}$ is a rational proximal contraction of the first and second kinds and $\mathcal{T}\left(\mathcal{A}_{0}\right) \subseteq \mathcal{B}_{0}$. Then all the hypotheses of the Theorem 3.3 are satisfied and $d(1, \mathcal{T}(1))=$ $d(\mathcal{A}, \mathcal{B})$. Clearly, the Theorem 3.2 is not applicable in this case.

Competing interests

The authors declare that they have no competing interests.

Authors' contributions

All authors contributed equally and significantly in writing this paper. All authors read and approved the final manuscript

\section{Author details}

${ }^{1}$ Department of Mathematics, Disha Institute of Management and Technology, Satya Vihar, Vidhansabha-Chandrakhuri Marg, Mandir Hasaud, Raipur, Chhattisgarh 492101, India. ${ }^{2}$ Department of Mathematics, Faculty of Science, King Mongkut's University of Technology Thonburi (KMUTT), Bangkok, 10140, Thailand. ${ }^{3}$ Dipartimento di Matematica e Informatica, Università degli Studi di Palermo, Via Archirafi 34, Palermo, 90123, Italy. 


\section{Acknowledgements}

The third author is supported by Università degli Studi di Palermo, Local University Project R. S. ex 60\%. The second author was supported by the Commission on Higher Education, the Thailand Research Fund, and the King Mongkuts University of Technology Thonburi (Grant No. MRG5580213).

\section{Received: 12 January 2013 Accepted: 26 March 2013 Published: 12 April 2013}

\section{References}

1. Eldred, A, Veeramani, PL: Existence and convergence of best proximity points. J. Math. Anal. Appl. 323, 1001-1006 (2006). doi:10.1016/j.jmaa.2005.10.081

2. Fan, K: Extensions of two fixed point theorems of F. E. Browder. Math. Z. 112, 234-240 (1969). doi:10.1007/BF01110225

3. Prolla, JB: Fixed point theorems for set valued mappings and existence of best approximations. Numer. Funct. Anal. Optim. 5, 449-455 (1982)

4. Reich, S: Approximate selections, best approximations, fixed points and invariant sets. J. Math. Anal. Appl. 62, 104-113 (1978). doi:10.1016/0022-247X(78)90222-6

5. Sehgal, VM, Singh, SP: A generalization to multifunctions of Fan's best approximation theorem. Proc. Am. Math. Soc. 102,534-537 (1988)

6. Sehgal, VM, Singh, SP: A theorem on best approximations. Numer. Funct. Anal. Optim. 10, 181-184 (1989) doi:10.1080/01630568908816298

7. Vetrivel, V, Veeramani, P, Bhattacharyya, P: Some extensions of Fan's best approximation theorem. Numer. Funct. Anal. Optim. 13, 397-402 (1992). doi:10.1080/01630569208816486

8. Al-Thagafi, MA, Shahzad, N: Best proximity sets and equilibrium pairs for a finite family of multimaps. Fixed Point Theory Appl. 2008, Article ID 457069 (2008)

9. Al-Thagafi, MA, Shahzad, N: Best proximity pairs and equilibrium pairs for Kakutani multimaps. Nonlinear Anal. 70(3), 1209-1216 (2009). doi:10.1016/j.na.2008.02.004

10. Al-Thagafi, MA, Shahzad, N: Convergence and existence results for best proximity points. Nonlinear Anal. 70(10), 3665-3671 (2009). doi:10.1016/j.na.2008.07.022

11. Anuradha, J, Veeramani, P: Proximal pointwise contraction. Topol. Appl. 156(18), 2942-2948 (2009). doi:10.1016/j.topol.2009.01.017

12. Balaganskii, VS, Vlasov, LP: The problem of the convexity of Chebyshev sets. Usp. Mat. Nauk 51, 125-188 (1996)

13. Banach, S: Sur les opérations dans les ensembles absraites et leurs applications. Fundam. Math. 3, 133-181 (1922)

14. Borodin, PA: An example of a bounded approximately compact set that is not compact. Russ. Math. Surv. 49, 153-154 (1994)

15. Di Bari, C, Suzuki, T, Vetro, C: Best proximity points for cyclic Meir-Keeler contractions. Nonlinear Anal. 69(11), 3790-3794 (2008). doi:10.1016/j.na.2007.10.014

16. Efimov, NV, Stechkin, SB: Approximative compactness and Chebyshev sets. Dokl. Akad. Nauk SSSR 140, $522-524$ (1961) (in Russian)

17. Eldred, A, Kirk, WA, Veeramani, P: Proximinal normal structure and relatively nonexpanisve mappings. Stud. Math. 171(3), 283-293 (2005). doi:10.4064/sm171-3-5

18. Karpagam, S, Agrawal, S: Best proximity point theorems for $p$-cyclic Meir-Keeler contractions. Fixed Point Theory Appl. 2009(9), Article ID 197308 (2009)

19. Kim, WK, Kum, S, Lee, KH: On general best proximity pairs and equilibrium pairs in free abstract economies. Nonlinear Anal. 68(8), 2216-2227 (2008). doi:10.1016/j.na.2007.01.057

20. Mongkolkeha, C, Kumam, P: Best proximity point theorems for generalized cyclic contractions in ordered metric spaces. J. Optim. Theory Appl. 155, 215-226 (2012). doi:10.1007/s10957-012-9991-y

21. Mongkolkeha, C, Kumam, P: Some common best proximity points for proximity commuting mappings. Optim. Lett. (2012) doi:10.1007/s11590-012-0525-1

22. Mongkolkeha, C, Cho, YJ, Kumam, P: Best proximity points for generalized proximal C-contraction mappings in metric spaces with partial orders. J. Inequal. Appl. 2013, 94 (2013). doi:10.1186/1029-242X-2013-94

23. Sadiq Basha, S: Extensions of Banach's contraction principle. Numer. Funct. Anal. Optim. 31, 569-576 (2010). doi:10.1080/01630563.2010.485713

24. Sadiq Basha, S: Best proximity points: global optimal approximate solution. J. Glob. Optim. (2010) doi:10.1007/s10898-009-9521-0

25. Sadiq Basha, S, Shahzad, N, Jeyaraj, R: Common best proximity points: global optimization of multi-objective functions. Appl. Math. Lett. 24, 883-886 (2011). doi:10.1016/j.aml.2010.12.043

26. Sadiq Basha, S, Veeramani, P: Best approximations and best proximity pairs. Acta Sci. Math. 63, $289-300$ (1997)

27. Sadiq Basha, S, Veeramani, P: Best proximity pair theorems for multifunctions with open fibres. J. Approx. Theory 103, 119-129 (2000). doi:10.1006/jath.1999.3415

28. Sadiq Basha, S, Veeramani, P, Pai, DV: Best proximity pair theorems. Indian J. Pure Appl. Math. 32, 1237-1246 (2001)

29. Sadiq Basha, S, Shahzad, N, Best proximity point theorems for generalized proximal contractions. Fixed Point Theory Appl. 2012, 42 (2012)

30. Sankar Raj, V, Veeramani, P: Best proximity pair theorems for relatively nonexpansive mappings. Appl. Gen. Topol. 10(1), 21-28 (2009)

31. Shahzad, N, Sadiq Basha, S, Jeyaraj, R: Common best proximity points: global optimal solutions. J. Optim. Theory Appl. 148, 69-78 (2011). doi:10.1007/s10957-010-9745-7

32. Sanhan, W, Mongkolkeha, C, Kumam, P: Generalized proximal $\psi$-contraction mappings and best proximity points. Abstr. Appl. Anal. 2012, Article ID 896912 (2012)

33. Sintunavarat, W, Kumam, K: Coupled best proximity point theorem in metric spaces. Fixed Point Theory Appl. 2012, 93 (2012)

34. Srinivasan, PS: Best proximity pair theorems. Acta Sci. Math. 67, 421-429 (2001)

35. Suzuki, T, Kikkawa, M, Vetro, C: The existence of best proximity points in metric spaces with the property UC. Nonlinear Anal. 71, 2918-2926 (2009). doi:10.1016/j.na.2009.01.173 
36. Suzuki, T, Vetro, C: Three existence theorems for weak contractions of Matkowski type. Int. J. Math. Stat. 6, 110-120 (2010).

37. Vetro, C: Best proximity points: convergence and existence theorems for p-cyclic mappings. Nonlinear Anal. 73 2283-2291 (2010). doi:10.1016/j.na.2010.06.008

38. Wlodarczyk, K, Plebaniak, R, Banach, A: Best proximity points for cyclic and noncyclic set-valued relatively quasiasymptotic contractions in uniform spaces. Nonlinear Anal. 70(9), 3332-3341 (2009). doi:10.1016/j.na.2008.04.037

39. Wlodarczyk, K, Plebaniak, R, Banach, A: Erratum to: best proximity points for cyclic and noncyclic set-valued relatively quasi-asymptotic contractions in uniform spaces. Nonlinear Anal. 71, 3585-3586 (2009). doi:10.1016/j.na.2008.11.020

40. Wlodarczyk, K, Plebaniak, R, Obczynski, C: Convergence theorems, best approximation and best proximity for set-valued dynamic systems of relatively quasi-asymptotic contractions in cone uniform spaces. Nonlinear Anal. 72, 794-805 (2010). doi:10.1016/j.na.2009.07.024

41. Kirk, WA, Reich, S, Veeramani, P: Proximinal retracts and best proximity pair theorems. Numer. Funct. Anal. Optim. 24 851-862 (2003). doi:10.1081/NFA-120026380

doi:10.1186/1687-1812-2013-95

Cite this article as: Nashine et al.: Best proximity point theorems for rational proximal contractions. Fixed Point Theory and Applications 2013 2013:95.

\section{Submit your manuscript to a SpringerOpen ${ }^{\circ}$ journal and benefit from:}

- Convenient online submission

- Rigorous peer review

- Immediate publication on acceptance

Open access: articles freely available online

- High visibility within the field

- Retaining the copyright to your article 